\title{
Michel Cordillot (dir.), La révolution de la machine à coudre
}

Les Cahiers d'Adiamos 89, $\mathrm{n}^{\circ}$ 16, mai 2018, 203 p.

\section{Fabien Knittel}

\section{(Q) OpenEdition \\ Journals}

\section{Édition électronique}

URL : http://journals.openedition.org/artefact/3737

DOI : $10.4000 /$ artefact.3737

ISSN : 2606-9245

\section{Éditeur :}

Association Artefact. Techniques histoire et sciences humaines, Presses universitaires du Midi

\section{Édition imprimée}

Date de publication : 15 mars 2019

Pagination : 359-362

ISBN : 978-2-8107-0623-5

ISSN : $2273-0753$

\section{Référence électronique}

Fabien Knittel, « Michel Cordillot (dir.), La révolution de la machine à coudre », Artefact [En ligne], 9 | 2018, mis en ligne le 04 mars 2020, consulté le 27 novembre 2020. URL : http://journals.openedition.org/ artefact/3737 ; DOI : https://doi.org/10.4000/artefact.3737

Ce document a été généré automatiquement le 27 novembre 2020.

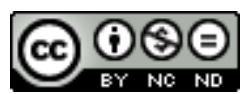

Artefact, Techniques, histoire et sciences humaines est mise à disposition selon les termes de la Licence Creative Commons Attribution - Pas d'Utilisation Commerciale - Pas de Modification 4.0 International. 


\section{Michel Cordillot (dir.), La révolution de la machine à coudre}

Les Cahiers d'Adiamos 89, n 16, mai 2018, 203 p.

\section{Fabien Knittel}

1 Ce recueil, coordonné par Michel Cordillot, et qui fait l'objet du numéro 16 de la revue Les Cahiers d'Adiamos 89, correspond aux actes du colloque organisé par l'association Adiamos 89 à Auxerre le 25 novembre 2017. Le sujet choisi, qui peut sembler de prime abord étonnant, ne l'est en réalité absolument pas tellement la machine à coudre occupe une place centrale dans l'univers des techniques du $\mathrm{XIX}^{\mathrm{e}}$ et du début du $\mathrm{Xx}^{\mathrm{e}}$ siècle.

2 Le recueil est organisé autour de huit articles de tailles assez inégales puisque l'article de Manuel Charpy, «Piqué-machine et cousu-main. Succès et rejets de la machine à coudre dans la confection au XIX siècle», ne compte pas moins de 45 pages (p. 67-112) tandis que l'article conclusif de Denis Martin, "Couturière d'aujourd'hui dans l'Yonne ", n'en compte que deux et demie (p.199-201). Nonobstant ces inégalités de longueur entre les articles, les textes sont tous à plusieurs titres, mais de manière différente, des plus intéressants. Le recueil possède son unité autour de son objet, la machine à coudre, mais chaque article est néanmoins indépendant des autres en ce sens que des répétitions fréquentes permettent de lire des textes qui se suffisent à euxmêmes. Pour les lecteurs ou lectrices intéressés par l'un ou l'autre texte c'est un avantage indéniable. En revanche, pour ceux ou celles qui souhaitent en faire une lecture entière et linéaire cela pourra sembler un peu fastidieux. Au-delà de la qualité intrinsèque de chaque article, l'ouvrage est richement illustré avec de nombreuses gravures, photographies et reproductions de publicités qui occupent quasiment un quart du volume. C'est d'un grand intérêt de compiler des documents iconographiques relevant de l'histoire des techniques, facilitant ainsi leur consultation. L'image accompagne le discours écrit: l'analyse de l'iconographie s'en trouve d'autant valorisée.

3 Dans son article introductif, intitulé «Genèse de la machine à coudre " (p.7-26), François Jarrige donne une définition de la machine à coudre : «il s'agit d'un appareil 
qui exécute mécaniquement les points de couture, le plus souvent en utilisant deux fils » (p. 7). Dès les premières pages, l'auteur, explique qu'étudier la machine à coudre est du plus grand intérêt pour l'histoire des techniques, certes, mais aussi pour l'histoire économique et sociale car c'est le premier objet mécanique fabriqué en grande série et vendu à l'échelle mondiale. Or, il précise aussi que l'objet en lui-même est encore mal connu des historiens et historiennes. Le but du recueil est donc de combler cette lacune historiographique. Les machines à coudre utilisées principalement par les femmes, mais pas seulement car les hommes les utilisent aussi dans certains ateliers mécanisés, sont un objet de recherche adéquat pour poser des questions sur les recompositions des identités de genre durant le $\mathrm{XIX}^{\mathrm{e}}$ et le début du $\mathrm{Xx}^{\mathrm{e}}$ siècle. Cette machine à coudre sert aussi à l'historien pour discuter des processus d'innovation. Suivent des développements sur les inventeurs, Barthélemy Thimonnier et Walter Hunt. Sauf que l'émergence de la machine à coudre est beaucoup plus complexe, à partir des années 1830, et il est bien difficile de décréter à qui revient le mérite de son invention stricto sensu. Mais est-ce bien là le plus intéressant ? Sans doute non. Ce qui se joue dans les querelles d'invention est bien plus captivant. Le XIX siècle est caractérisé par la mise au point de machines permettant de coudre avec des points particuliers comme le point de chaînette de Thimonnier, le point de navette de Hunt ou alors le point noué d'Elias Howe. Le cas Thimonnier est développé par Éric Perrin dans son article intitulé «Naissance et Renaissance de la machine à coudre à Saint-Étienne. De Barthélémy Thimonnier à Étienne Mimard»(p.27-65). L'auteur explique les premiers pas de Thimonnier à Saint-Étienne au début des années 1830 puis « l'éclipse » de la production de machines à coudre dans la ville avant son retour, pour une " seconde phase » (p. 38), au sein de la Manufacture française d'armes et cycles, grâce à Étienne Mimard, presque un siècle plus tard. L'ensemble du recueil est structuré sur l'alternance d'études de cas localisées, Saint-Étienne ou Auxerre (Jean-Charles Guillaume, "Les couturières à Auxerre du Second Empire à la Seconde Guerre mondiale », p. 139-171), et des articles de portée plus générale.

4 La machine à coudre comme de nombreuses autres inventions n'est donc pas mise au point une bonne fois pour toutes par un unique inventeur à un moment précis. L'élaboration et les améliorations des machines qui deviennent progressivement les machines à coudre telle qu'elles sont vendues à partir de la seconde moitié du XIX siècle, s'effectuent peu à peu durant les trois décennies de 1830 à 1860. D'ailleurs, il faut attendre le mois d'août 1851 pour qu'Isaac Merrit Singer dépose un brevet et fonde une des premières compagnies qui commercialisent à grande échelle des machines à coudre.

5 L'équipement des ménages est assez rapide. Vers 1890, indique François Jarrige, 80 \% des foyers en France sont équipés d'au moins une machine à coudre. C'est dire son succès populaire. Celui-ci est dû à la puissance commerciale et industrielle de la firme Singer ainsi qu'au recours massif à la publicité (de très nombreuses affiches et autres encarts de catalogues de vente sont répertoriés dans le volume, notamment p. 50-63). Jean-Charles Guillaume consacre un article aux réclames vantant les machines à coudre à Auxerre durant un siècle, de 1865 à 1960 (p. 177-198). Rapidement la production des machines à coudre devient une production de masse, en série avec des pièces standardisées favorisant et la production et les réparations. En France, c'est Peugeot qui domine la fabrication durant les années 1870 et teste l'usage de la vapeur pour les 
faire fonctionner. Plus tard, durant les années 1920, l'électrification se généralise transformant les modalités d'utilisation des machines.

Manuel Charpy explique cependant la méfiance des consommateurs pour les vêtements confectionnés à la machine. Beaucoup, au début du xxe siècle, jugent que le cousu-main garanti une meilleure qualité de vêtement. Dans la lignée d'Edgerton (« De l'innovation aux usages. Dix thèses éclectiques sur l'histoire des techniques", Annales Histoire. Sciences Sociales, juillet-octobre $\left.1998, \mathrm{n}^{\circ} 4-5\right)$ et de ses thèses sur l'histoire des techniques, Manuel Charpy illustre la persistance du cousu-main, ancienne technique qui résiste face au développement et au succès de la nouvelle technique, la couture à la machine. C'est pourquoi le choix du terme "révolution " pour le titre de l'ouvrage est discutable. Il s'agit davantage de transformations sur un temps moyen à long que d'un brusque changement radical.

De son côté Michel Cordillot s'interroge sur les réactions du monde ouvrier face à la mécanisation de la couture (p. 113-137) et concède qu'y répondre simplement est loin d'être facile. Beaucoup d'auteurs relèvent aussi les préoccupations des médecins au sujet des possibles pathologies ou de l'amoralité en lien avec l'usage de la machine à coudre par des femmes. Enfin, Michel Cordillot propose aussi un court texte consacré à «La machine à coudre et le Cinématographe des frères Lumière "(p. 173-175). Pour élaborer leur Cinématographe, les frères Lumière s'inspirent techniquement du fonctionnement de la machine à coudre, notamment la griffe qui « fait successivement s'avancer puis s'immobiliser le tissu le temps que le point soit réalisé » (p. 174). Louis Lumière imagine alors, puis met au point, un système proche qui permet de «faire défiler la pellicule et l'immobiliser un vingt-cinquième de seconde devant la fenêtre de projection » (p. 174).

8 Au final ce recueil, malgré quelques coquilles d'impression et ses quelques répétitions, est d'une grande richesse et la somme d'informations contenues en à peine 200 pages en fait d'ores et déjà un outil clé pour la connaissance de l'histoire des machines à coudre, pan immense de l'historiographie des techniques mais aussi économique, sociale et culturelle. Objet d'histoire totale que cette machine à coudre : si ce concept n'était pas quelque peu vieilli et n'avait pas montré autant ses limites nous serions presque tentés de l'écrire. On s'en approche en tout cas. Lecture recommandée.

\section{AUTEUR}

\section{FABIEN KNITTEL}

Université de Bourgogne-Franche-Comté (UFC), Centre Lucien Febvre, EA 2273 et LHSP-Archives Poincaré, UMR 7117 